\title{
The CIpXP and CIpAP proteases degrade proteins with carboxy-terminal peptide tails added by the SsrA-tagging system
}

\author{
Susan Gottesman, ${ }^{1,3}$ Eric Roche, ${ }^{2}$ YanNing Zhou, ${ }^{1}$ and Robert T. Sauer ${ }^{2}$ \\ ${ }^{1}$ Laboratory of M olecular Biology, N ational Cancer Institute, Bethesda, Maryland 20892 USA; ${ }^{2}$ Department of Biology, \\ Massachusetts Institute of Technology, Cambridge, M asaschusetts 02139 USA
}

\begin{abstract}
Intemuption of translation in Escherichia coli can lead to the addition of an 11-residue carboxy-terminal peptide tail to the nascent chain. This modification is mediated by SsrA RNA (also called 10Sa RNA and tmRNA) and marks the tagged polypeptide for proteolysis. Degradation in vivo of $\lambda$ repressor amino-terminal domain variants bearing this carboxy-terminal SsrA peptide tag is shown here to depend on the cytoplasmic proteases CIpXP and CIPAP. Degradation in vitro of SsrA-tagged substrates was reproduced with purified components and required a substrate with a wild-type SsrA tail, the presence of both CIpP and either ClpA or CIpX, and ATP. Clp-dependent proteolysis accounts for most degradation of SsrA-tagged amino-domain substrates at $32^{\circ} \mathrm{C}$, but additional proteases contribute to the degradation of some of these SsrA-tagged substrates at $39^{\circ} \mathrm{C}$. The existence of multiple cytoplasmic proteases that function in SsrA quality-control surveillance suggests that the SsrA tag is designed to serve as a relatively promiscuous signal for proteolysis. Having diverse degradation systems able to recognize this tag may increase degradation capacity, permit degradation of a wide variety of different tagged proteins, or allow SsrA-tagged proteins to be degraded under different growth conditions.
\end{abstract}

[Key Words: 10Sa RNA; $\lambda$ repressor; Escherichia coli; intracellular proteolysis; molecular recognition; ATP-dependent degradation]

Received January 13, 1998; revised version accepted March 2, 1998.

Certain proteins and protein fragments in Escherichia coli are modified by carboxy-terminal addition of an 11residue peptide tag (Tu et al. 1995). This tagging process requires functional SsrA RNA (10Sa RNA), which encodes the last 10 residues of the peptide ( $T u$ et al. 1995) and results in rapid degradation of the tagged protein by carboxy-terminal-specific proteases (Keiler et al. 1996). SsrA-mediated tagging of proteins transl ated from defective messenger RNAs lacking termination codons has been demonstrated, and a model in which SsrA functions both as a tRN $A$ and an mRN A has been proposed (Keiler et al. 1996). The 363-nucleotide SsrA RNA has sequences that form a tRN A-like structure and has been shown to be chargeable with alanine (Komine et al. 1994; Williams and Bartel 1996; Felden et al. 1997). In the model proposed by Keiler et al., when ribosomes stall at the $3^{\prime}$ end of the damaged message, SsrA charged with alanine binds to the ribosome like a tRN A and contributes the alanine to the idle nascent chain. Translation then switches from the mRN A to a small open reading frame (ORF) in SsrA that encodes the carboxy-terminal

${ }^{3}$ Corresponding author.

E-MAIL susang@helix.nih.gov; FAX (301) 496-3875. degradation peptide. This system provides both a method to avoid the accumulation of ribosomes stalled at the end of defective messages and a general qualitycontrol mechanism that allows the cell to rid itself of incomplete protein fragments that might have inappropriate cellular activities. Cells devoid of SsrA RNA grow more slowly and show a certain degree of temperature sensitivity (Oh and Apirion 1991; Komine et al. 1994; Trempy et al. 1994).

The involvement of carboxy-terminal amino-acid sequences in targeting proteins for rapid degradation was recognized before the discovery of the SsrA-tagging system (Bowie and Sauer 1989; Parsell et al. 1990), and a periplasmic protease (Tsp or Prc) that degrades protein substrates in a carboxy-terminal-specific manner was purified and characterized (Silber et al. 1992). The carboxy-terminal substrate sequences recognized by Tsp are similar to those of the SsrA tag (Keiler et al. 1995; Tu et al. 1995), and T sp is responsible for degradation of SsrAtagged proteins that are exported to the periplasm (Keiler et al. 1996). Cytoplasmic proteins with carboxy-terminal degradation sequences, however, are still proteolyzed rapidly in cells lacking T sp (Silber and Sauer 1994; Keiler et al. 1996), indicating that other proteases must be re- 
sponsible for carboxy-terminal-specific degradation of proteins in the bacterial cytoplasm.

Essentially all cytoplasmic degradation in prokaryotes, archaea, and eukaryotes is energy-dependent. E. coli, for example, has at least five ATP-dependent proteases [Lon (La); HflB (FtsH); CIpAP; CIpXP; and CIpYQ (HsIUV)] (for review, see Gottesman 1996). These enzymes appear to have distinct substrate preferences, as a mutation in a single protease gene is often sufficient to stabilize a specific unstable protein. For example, mutations in Lon lead to stabilization of the $N$ protein of bacteriophage $\lambda$, the SulA and RcsA proteins of E. coli, and the CcdA protein of the episomal $F$ factor. HflB appears to be responsible for degradation of the cll protein of $\lambda$ and the heat-shock $\sigma$ factor RpoH (Herman et al. 1993, 1995). The principal substrates for CIpYQ degradation have not yet been identified, although this two-component protease has been implicated in degradation of both Lon subtsrates and HfIB substrates in vivo (Missiakas et al. 1996; Kanemori et al. 1997; Khattar 1997; W.-F. Wu and S. Gottesman, unpubl.). CIpAP and CIpXP are two-component proteases that share a common proteolytic subunit, CIpP, but have different ATPase regulatory subunits, CIpA or CIpX. Proteins stabilized by mutations in $\mathrm{clpX}$ but not in clpA include $\lambda \mathrm{O}$, phage $\mathrm{Mu}$ repressor variants, and the stationary-phase $\sigma$ factor, RpoS; clpA but not clpX mutants stabilize certain LacZ fusion proteins and the MazE protein. CIpB, an ATPase with extensive sequence similarity to $\mathrm{ClpA}$, has not thus far been demonstrated to have a direct role in proteolysis but may act as a chaperone (Squires and Squires 1992).

In the studies presented here, we show that intracellular degradation of variants of the amino-terminal domain of $\lambda$ repressor containing the SsrA peptide tag is dramatically reduced in cells lacking CIpP or lacking both $\mathrm{CIpX}$ and $\mathrm{CI} \mathrm{pA}$, and is somewhat reduced in cells lacking CIpX or CIpA only. Purified CIpXP and purified CIpAP degrade SsrA-tagged protein substrates in vitro, suggesting that these ATP-dependent enzymes are directly responsi ble for degradation of SsrA-tagged proteins in the bacterial cytoplasm.

\section{Results}

At sufficiently high concentrations, a protein containing the 93 amino-acid amino-terminal domain of $\lambda \mathrm{cl}$ repressor binds to the $\lambda$ operators and prevents lytic growth of superinfecting phage (Sauer et al. 1979; Jordan and Pabo 1988). When degradation signals such as the SsrA tag, however, are attached to the carboxyl terminus of this fragment, proteolysis reduces the steady-state quantities of this domain to levels insufficient for function (Parsell et al. 1990; Keiler et al. 1996). Therefore, phage immunity provides an assay for the intracellular degradation of tagged amino-terminal domain derivatives.

For the work reported here, we have used three pai rs of amino-domain variants (see Fig. 1). In each pair, one variant contains the protease-sensitive SsrA tag (AANDENYALAA) and the other bears a protease-resistant control tag (AAN DEN YALDD); the pairs differ in the linker regions that connect these carboxy-terminal tags to the amino-terminal domain. We have al so used two phages, $\lambda \mathrm{Cl}^{-}$, which produces no functional $\lambda$ repressor, and the weaker $\lambda$ c17 (see Materials and Methods), which is capable of producing some $\lambda$ repressor. Wild-type strains expressing the three SsrA-tagged proteins $(\lambda N$-AA, $\lambda N$ L1-AA, and $\lambda N$-L2-AA) were found, as expected, to be sensitive to superinfection by $\lambda \mathrm{Cl}^{-}$or $\lambda \mathrm{c} 17$, whereas cells expressing the three control-tagged proteins $(\lambda N-D D$, $\lambda \mathrm{N}$-L1-DD, and $\lambda \mathrm{N}$-L2-DD) were immune.

Immunity in protease-deficient mutants

To test for cytoplasmic proteases required for degradation of SsrA-tagged proteins, we assayed for increased $\lambda$ immunity in known or suspected protease-defective

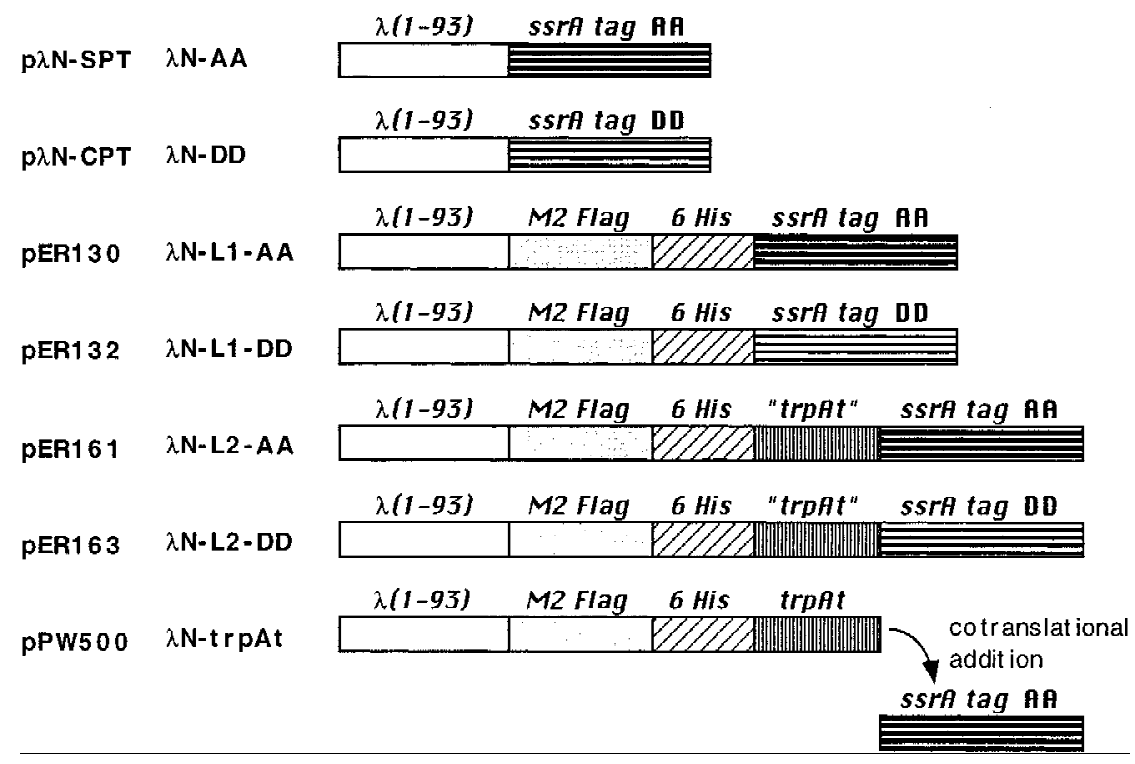

Figure 1. Proteins used for degradation studies of SsrA-tagged proteins. Cartoon representation of the structures of hybrid proteins. The names of these proteins and of the plasmids that encode them are indicated to the left. $[\lambda(1-93)]$ The 93-residue sequence of the amino-terminal, operatorbinding domain of the $\lambda \mathrm{cl}$ repressor. (M2 Flag) The epitope sequence DYKDDDDK. (6His) The sequence $\mathrm{HHHHHH}$. (SsrA tag AA) The sequence AAN DENY ALAA; (SsrA tag DD) The sequence AANDENYALDD. (trpAt) The AARLMSG sequence encoded by the stem-loop region of the trpAt transcriptional terminator. Note that because of heterogeneity in the position of SsrA tagging (Keiler et al. 1996), the $\lambda \mathrm{N}$-trpAt protein may consist of several species, some of which differ by 1 or 2 amino acids from the otherwise identical sequence of $\lambda N$-L2-AA. 


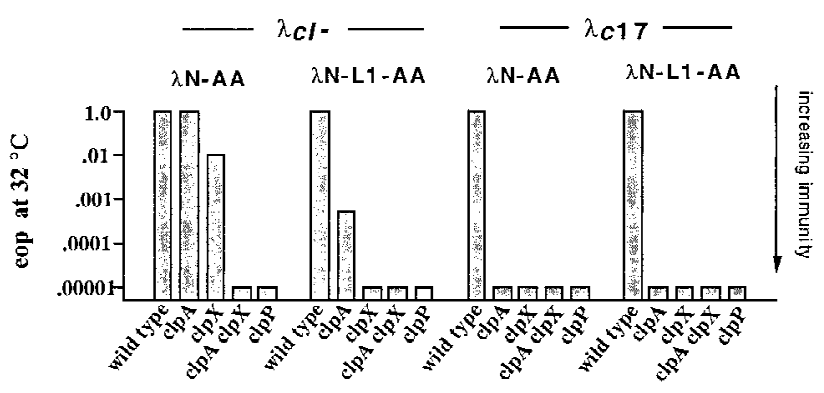

Figure 2. $\lambda N-A A$ and $\lambda N-L 1-A A$ mediated immunity in $\mathrm{clp}$ mutants. Isogenic strains expressing $\lambda N$-AA or $\lambda N$-L1-AA were grown in tryptone broth with ampicillin and plated on LB-Amp plates in top agar. Ten-microliter serial dilutions of $\lambda \mathrm{Cl}^{-}$or $\lambda \mathrm{cl} 7$ phage were spotted, plates were incubated overnight at $32^{\circ} \mathrm{C}$, and an approximate eop was cal culated rel ative to plating on the wild-type strain SG22163 transformed with pBR322. E. coli strains: wild-type (SG22163); clpP (SG22174); clpA (SG22176); clpX SG22177); and clpAclpX (SG22178). In control experiments, the heteroimmune phage $\lambda \mathrm{imm} 21 \mathrm{cl}$, whose operators are not repressed by amino-terminal domain variants of $\lambda$ repressor, plated with an eop of 1 on all strains containing $\lambda N$-AA or $\lambda \mathrm{N}-\mathrm{L} 1-\mathrm{AA}$ and on wild-type strains expressing $\lambda \mathrm{N}-\mathrm{DD}$ or $\lambda \mathrm{N}$-L1-DD. $\lambda \mathrm{Cl}^{-}$and $\lambda \mathrm{Cl}$ (7) plated with an eop of $10^{-4}$ or less on wild-type strains expressing $\lambda \mathrm{N}-\mathrm{DD}$ or $\lambda \mathrm{N}-\mathrm{L1}-\mathrm{DD}$.

strains expressing the $\lambda \mathrm{N}-\mathrm{AA}$ or $\lambda \mathrm{N}$-L1-AA proteins. No changes in immunity were observed in strains with an insertion mutation in $\mathrm{ClpB}$, an insertion mutation in hflA, a deletion of Ion, mutations in Ion and $\mathrm{ClpQ}$, or a temperature-sensitive mutation in hflB (data not shown). A very different result was observed in cells with defects in the CIpXP or CIpAP proteases. In strains containing insertions in the protease subunit (clpP) or regulatory subunits (clpA or $\mathrm{clpX}$ ), expression of $\lambda \mathrm{N}-\mathrm{AA}$ or $\lambda \mathrm{N}$-L1-AA resulted in immunity to superinfection by $\lambda \mathrm{c} 17$ (Fig. 2), suggesting that these proteases are involved in degradation of SsrA-tagged proteins.

Among the different clp mutants, no differences in $\lambda c 17$ immunity were observed, but clear differences in immunity to $\lambda \mathrm{Cl}^{-}$were evident (Fig. 2). The patterns of $\lambda \mathrm{Cl}^{-}$immunity suggest that the highest steady-state levels of SsrA-tagged amino-domain proteins are achieved when CIpP is defective or when both CIpX and CIpA are inactivated by mutation. In contrast, inactivation of $\mathrm{ClpX}$ alone in a strain expressing $\lambda \mathrm{N}-\mathrm{AA}$ resulted in very modest immunity to $\lambda \mathrm{Cl}^{-}$, and inactivation of ClpA alone gave partial immunity ( $\lambda \mathrm{N}$-L1-AA) or no immunity $\left(\lambda \mathrm{N}-\mathrm{AA}\right.$ ) to $\mathrm{\lambda Cl}^{-}$(see Fig. 2 for quantitation of immunity). In control experiments, we found that expression of the control-tagged $\lambda N$-DD or $\lambda N$-L1-DD proteins provided full immunity [Efficiencies of plating (eop) $10^{-4}$ or lower] to $\lambda \mathrm{Cl}^{-}$and $\lambda \mathrm{c} 17$, in both wild-type and $\mathrm{clp}$ mutants. Therefore, the Clp-mediated increases in phage immunity are dependent on the presence of $\lambda$ aminoterminal domain proteins bearing a wild-type SsrA degradation tag at their carboxyl terminus. We interpret these data as suggesting that either CIpXP or CIpA P can degrade and thereby reduce the intracellular levels of the $\lambda \mathrm{N}-\mathrm{AA}$ and $\lambda \mathrm{N}-\mathrm{L} 1-\mathrm{AA}$ proteins.

\section{Protein turnover in vivo}

Degradation of $\lambda N$-AA or $\lambda N$-L1-AA in wild-type and protease-deficient strains was assayed directly by blocking protein synthesis and measuring the di sappearance of the protein by Western blotting. Transformed cells expressing either $\lambda \mathrm{N}-\mathrm{AA}$ or $\lambda \mathrm{N}$-L1-AA were grown, induced by the addition of IPTG for $30 \mathrm{~min}$, and treated with spectinomycin to block further protein synthesis. Samples were removed during the subsequent incubation and examined by Western blotting for remaining levels of $\lambda N$-AA or $\lambda N$-L1-AA. Figure $3 A$ shows a Western blot of $\lambda \mathrm{N}$-AA turnover in wild-type cells, in which the half-life is $<10 \mathrm{~min}$, and in clpP mutant cells, in which the half life is longer than $1 \mathrm{hr}$. The initial level of $\lambda \mathrm{N}-\mathrm{AA}$ al so was higher in the cl pP mutant host, a result consistent with the immunity experiments. In parallel experiments, the $\lambda N$-DD protein showed no detectable turnover (half life $>1 \mathrm{hr}$ ) in either the wild-type or clpP strain (data not shown). The rapid turnover of $\lambda \mathrm{N}-\mathrm{AA}$ and sl ow turnover of $\lambda N$-DD had been demonstrated previously in a different wild-type strain background (Keiler et al. 1996).

\section{A Chase Time (min)} $\begin{array}{llllllllll}0 & 5 & 10 & 20 & 30 & 45 & 60 & 0 & 30 & 60\end{array}$
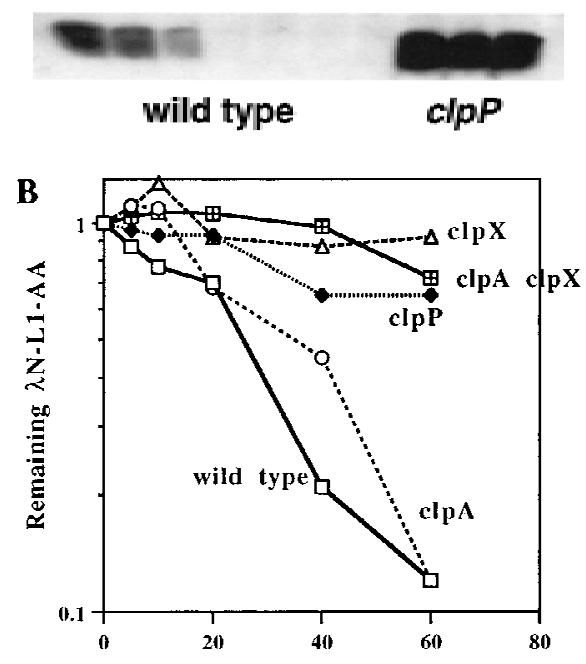

Chase Time (min)

Figure 3. Clp-dependent turnover in vivo of $\lambda N-A A$ and $\lambda N$ L1-AA. (A) Western blot analysis of $\lambda \mathrm{N}-\mathrm{AA}$ in wild-type (SG22163) or clpP mutant (SG22175) cells after spectinomycin blocking of protein synthesis. Cells were grown to mid-log phase at $32^{\circ} \mathrm{C}$ and induced with IPTG for 30 min before addition of spectinomycin. Samples were removed at the times shown into cold TCA, precipitated, and resuspended for electrophoresis in $15 \%$ SDS-polyacrylamide gels. Gels were blotted and probed with anti- $\lambda$-repressor antibody. (B) Turnover of $\lambda \mathrm{N}$-L1AA in wild-type and clp mutants. Experimental conditions were the same as in A except 10\%-20\% tricine SDS-polyacrylamide gels were used, and Western blots were probed with the M2 anti-FLAG antibody and quantified by the Eagle Eye II gel imaging system. Curves and strains are marked as for Fig. 2. 
Figure 3B shows time courses for $\lambda \mathrm{N}$-L1-AA degradation in a set of isogenic clp mutants. As expected from the immunity results, degradation of $\lambda \mathrm{N}$-L1-AA is fastest in the wild-type strain that contains functional CIpAP and CIpXP. A small increase in half life was observed in the CIpA-defective strain, and relatively long half lives were observed in strains lacking CIpP, CIpA and $\mathrm{ClpX}$, or ClpX. We note that in different experiments, the results with clpX mutant hosts were somewhat variable, often showing less stabilization than observed in Figure $3 \mathrm{~B}$; we currently have no explanation for this variability. M utations in Ion, $\mathrm{Cl} \mathrm{pB}$, hflA, or fhflB had no detectable effect on $\lambda \mathrm{N}$-LI-AA turnover under the conditions of these experiments (data not shown). Therefore, the high turnover rates of the $\lambda \mathrm{N}-\mathrm{AA}$ and $\lambda \mathrm{N}$-L1-AA proteins are entirely consistent with the immunity tests. More importantly, the turnover experiments demonstrate that the increased phage immunity and increased steady-state levels of $\lambda \mathrm{N}-\mathrm{AA}$ and $\lambda \mathrm{N}$-L1AA in CIpAP-defective or CIpXP-defective strains result mainly from decreased rates of degradation.

\section{Protein degradation in vitro}

To test directly for degradation of SsrA-tagged proteins by CIp proteases, ${ }^{35}$ S-labeled $\lambda N$-L1-AA and $\lambda N$-L1-DD were purified and incubated with purified CIpAP or ClpXP. As shown in Figure 4A, both Clp proteases degraded the SsrA-tagged $\lambda N$-L1-AA protein efficiently but degraded the control-tagged $\lambda \mathrm{N}$-L1-DD protein at rates only $5 \%$ to $10 \%$ of that seen with $\lambda \mathrm{N}$-L1-AA (Fig. 4A). Degradation required both $\mathrm{Cl} p \mathrm{pP}$ and either $\mathrm{CI} \mathrm{pX}$ or CIpA and, in every case, requi red ATP (data not shown). These results demonstrate that both CIPXP and CIPAP can degrade proteins with the carboxy-terminal SsrA degradation peptide.

As shown in Figure 4B, $\lambda N$-L1-AA and $\lambda N$-L1-DD were equally sensitive to degradation by chymotrypsin, a relatively nonspecific protease (Benyon and Bond 1989), indicating that the SsrA tag does not increase the general protease sensitivity of proteins to which it is attached. Purified $\lambda N$-L1-AA and $\lambda N$-L1-DD also had superimposible CD spectra (data not shown) and identical melting profiles in thermal denaturation experiments monitored by changes in circular dichroism (Fig. 4C). These data show that the susceptibility of $\lambda \mathrm{N}$-L1-AA to degradation by CIpXP and CIpAP does not result from major changes in the structure or stability of this tagged protein. Together, these results support a model in which the SsrA tag is recognized directly by CIpXP or CIpAP and argue against models in which the tag destabilizes the substrate, allowing proteases to recognize aminoacid sequences exposed in the denatured state.

\section{Degradation after SsrA tagging in vivo}

The experiments described above use amino-terminal domain variants in which the SsrA peptide tag is encoded by the genes for the $\lambda \mathrm{N}$-AA or $\lambda \mathrm{N}$-L1-AA proteins. Using the $\lambda \mathrm{N}$-trpAt system of Keiler et al. (1996), we
A

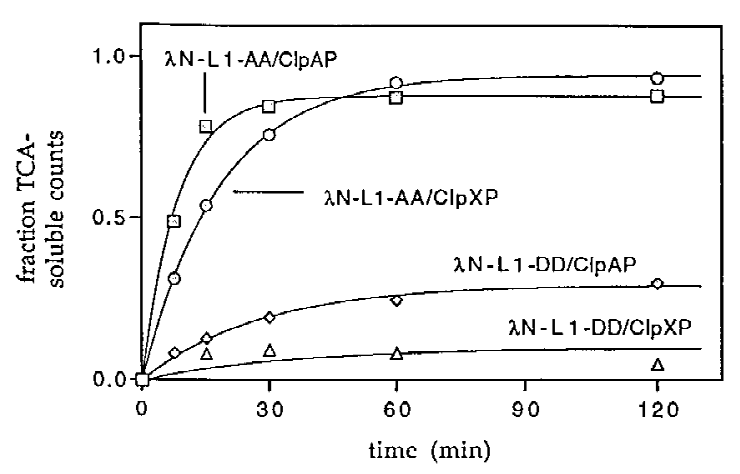

B

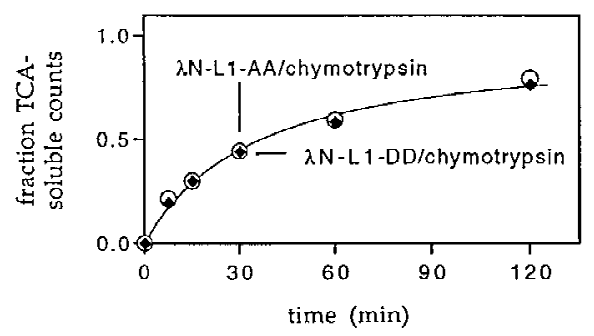

C

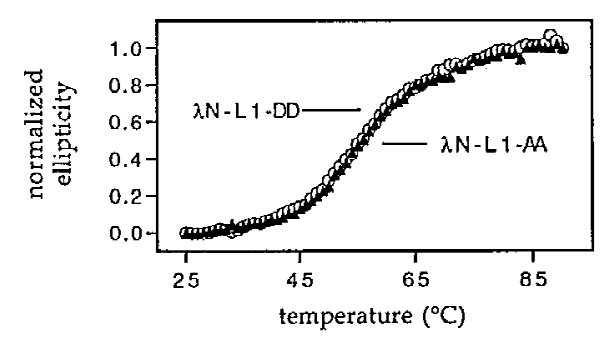

Figure 4. Degradation in vitro of SsrA-tagged substrates by CIpAP and CIpXP. (A) Purified ${ }^{35}$ S-labeled $\lambda N$-L1-AA or $\lambda N$-L1DD proteins were incubated with purified CIpAP or CIpXP in the presence of ATP at $30^{\circ} \mathrm{C}$ as described in $M$ aterials and $M$ ethods. Time-dependent release of TCA soluble counts is shown. (B) ${ }^{35}$ S-Labeled $\lambda N$-L1-AA or $\lambda N$-L1-DD were incubated with chymotrypsin using the same experimental conditions as in A. (C) Denaturation of the $\lambda \mathrm{N}$-LI-AA and $\lambda \mathrm{N}$-L1-DD proteins monitored by changes in circular dichroism shows no difference in thermal stability. $\mathrm{N}$ ormalized CD ellipticity was calculated as $\left(\epsilon_{25}-\epsilon\right) /\left(\epsilon_{25}-\epsilon 93\right)$ where e is the ellipticity at $230 \mathrm{~nm}$ at each temperature and $\epsilon_{25}$ and $\epsilon_{93}$ are the ellipticities at $230 \mathrm{~nm}$ at $25^{\circ} \mathrm{C}$ and $93^{\circ} \mathrm{C}$, respectively.

also examined turnover in vivo of proteins in which the carboxy-terminal degradation peptide is added cotranslationally by the SsrA-mediated tagging system. As diagrammed in Figure 1 , the gene for $\lambda \mathrm{N}$-trpAt encodes the amino-terminal domain and amino acids from the trpAt transcription terminator. There is no translation termination codon within this sequence. Therefore, when this message is read, translation ceases without termination and release of the growing polypeptide. Keiler et al. (1996) demonstrated that, under these circumstances, SsrA has an essential role in tagging and all ows rel ease of the stalled polypeptide. The $\lambda N-L 2-A A$ and $\lambda N$-L2-DD proteins contain the same amino acids from the trpAt 
terminator region but, unlike $\lambda \mathrm{N}$-trpAt, have the SsrA tag or control tag encoded in the gene (Fig. 1), and therefore are SsrA-independent.

Turnover experiments for $\lambda \mathrm{N}$-trpAt and $\lambda \mathrm{N}$-L2-AA are shown in Figure 5. $\lambda \mathrm{N}$-trpAt protein, expressed in an ssrA mutant, migrated faster in the gel (as expected for untagged protein) and was proteolytical ly stable (Fig. 5A; see al so Keiler et al. 1996). In the ssrA ${ }^{+}$host, only about half of the $\lambda \mathrm{N}$-trpAt protein appears to be tagged, but this species was degraded rapidly in the $\mathrm{clpP}^{+}$strain and had a longer half life in the $\mathrm{ClpP}^{-}$mutant (Fig. 5A). The shorter $\lambda \mathrm{N}$-trpAt protein in the ssrA ${ }^{+}$host appears to be untagged, based on its electrophoretic mobility, and was longer lived than the tagged protein. $\lambda \mathrm{N}-\mathrm{L} 2-\mathrm{AA}$, which has essentially the same sequence as tagged $\lambda \mathrm{N}$-trpAt, was turned over rapidl y in $\mathrm{ClpP}^{+}$cells and is significantly stabilized in the $\mathrm{ClpP}^{-}$mutant (Fig. 5B). These results show that SsrA-tagged $\lambda N$-trpAt and $\lambda N$-L2-AA are degraded in a clpP-dependent fashion. We note, however, that SsrA-tagged $\lambda \mathrm{N}$-trpAt was degraded faster than $\lambda \mathrm{N}$ L2-AA in both $\mathrm{ClpP}^{+}$and $\mathrm{ClpP}^{-}$strains (Fig. $5 \mathrm{C}$ ), indicating that the cotranslational, SsrA-dependent tagging of $\lambda \mathrm{N}$-trpAt may result in the tagged protein becoming exposed to or made more sensitive to other proteases in addition to being substrates for CIPAP and CIpXP.

\section{High-temperature effects on Clp-dependent turnover}

The immunity and turnover experiments described above were performed at $32^{\circ} \mathrm{C}$. We observed, however, that cel Is expressing $\lambda \mathrm{N}$-AA did not acquire immunity at $39^{\circ} \mathrm{C}$ in $\mathrm{clpP}$ or clpA clpX mutant strains (Fig. 6). Preliminary turnover experiments indicated that, as above, this lack of immunity reflected rapid degradation of the $\lambda \mathrm{N}$-AA protein at el evated temperatures, suggesting that proteases other than CIPAP or CIpXP must partici pate in degradation of this SsrA-tailed protein at $39^{\circ} \mathrm{C}$. C. Herman and R. D'Ari (pers. comm.) have made similar observations and identified $\mathrm{HFlB}$ as a protease contributing to $\lambda \mathrm{N}-\mathrm{AA}$ degradation under these conditions. Unlike $\lambda \mathrm{N}-\mathrm{AA}$, however, we found that $\lambda \mathrm{N}$-L1-AA was still immune in $\mathrm{clpP}$ or clpA clpX mutant strains at high temperature (Fig. 6), suggesting that $\lambda N$-L1-AA is not sensitive to $\mathrm{HflB}$ or proteases other than CIpXP or CIpAP at $39^{\circ} \mathrm{C}$. Because both of these proteins have the same SsrA tag but only $\lambda \mathrm{N}-\mathrm{AA}$ appears to be degraded in a CIpindependent fashion at $39^{\circ} \mathrm{C}$, factors in addition to the SsrA tag must influence degradation by proteases such as HflB.

\section{Discussion}

SsrA-mediated addition of a peptide signal to the carboxyl terminus of incompletely translated protein fragments is a newly discovered way for bacterial cells to mark abnormal proteins for destruction (Keiler et al. 1996). Cytoplasmic proteases that recognize and degrade SsrA-tagged proteins are critical components of this quality-control system and have now been identified.
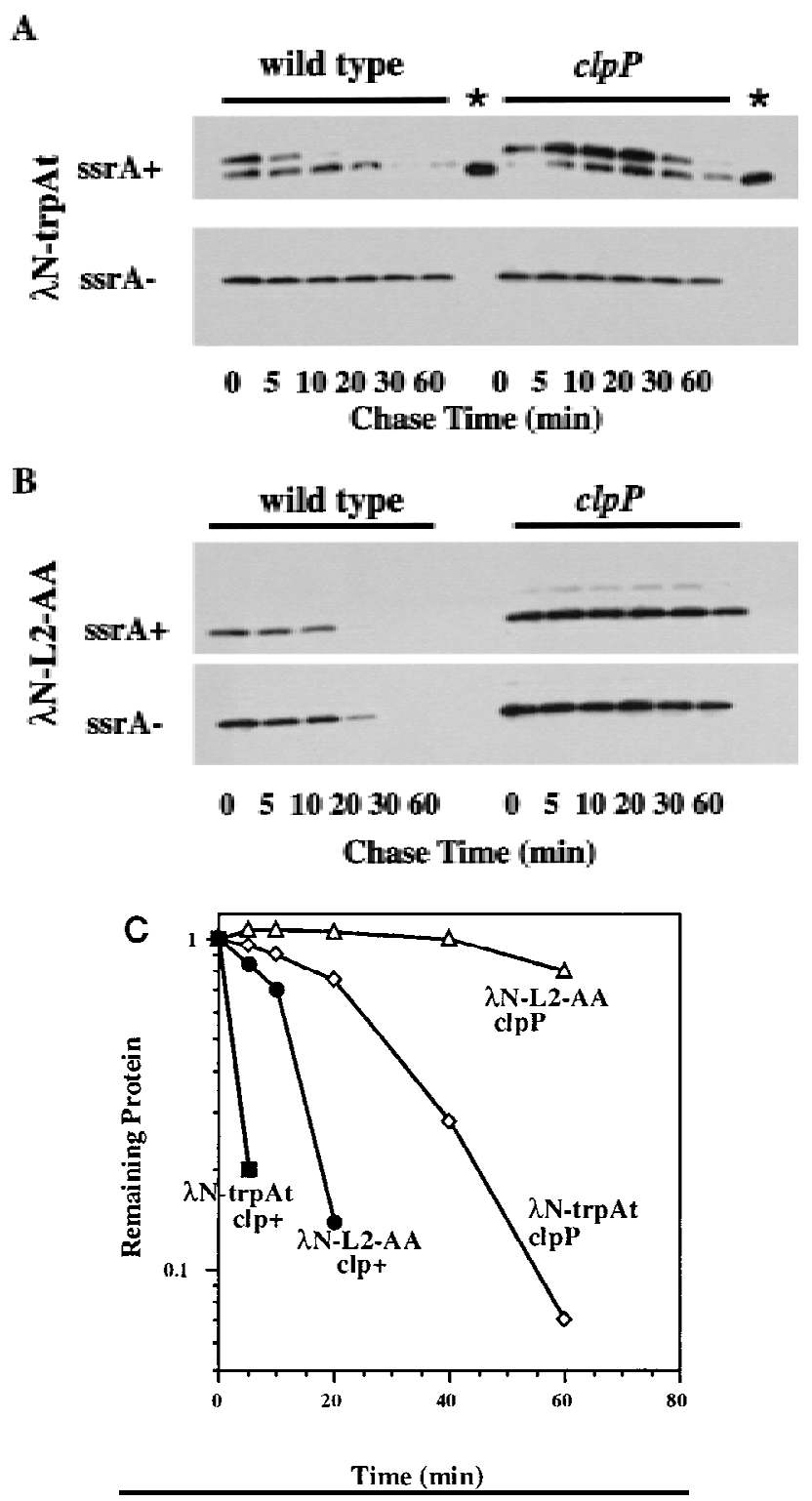

Figure 5. Degradation of $\lambda N$-trpAt and $\lambda N-L 2-A A$ in vivo. (A) Western blots of $\lambda \mathrm{N}$-trpAt turnover following spectinomycin treatment in $\mathrm{SSrA}^{+} \mathrm{Clp}^{+}$(SG22163), SsrA ${ }^{+} \mathrm{clpP}:: k a n$ (SG22175), ssrA::cat $\mathrm{clp}^{+}$(SG22183), and ssrA::cat clpP::kan (SG22184) strains. $N$ ote the presence of tagged and untagged variants of the $\lambda \mathrm{N}$-trpAt protein in ssrA ${ }^{+}$strains. Lanes marked with an asterisk are untagged samples from the ssrA- host run on the same gel as ssrA ${ }^{+}$samples to al low comparison of el ectrophoretic mobilities. Experimental procedures were the same as described in Fig. 3; $10 \%-20 \%$ gradient tricine gels ( $\mathrm{N}$ ovex) were used for el ectrophoresis. (B) Western blots of $\lambda \mathrm{N}$-L2-AA turnover. Strains and conditions were identical to those shown in A. In control experiments, $\lambda \mathrm{N}$-L2-DD expressed in the wild-type strain (SG22163) showed no significant turnover over $1 \mathrm{hr}$ (data not shown). (C) Time courses of degradation of SsrA-tagged $\lambda N$ trpAt protein (upper band in A) and $\lambda \mathrm{N}-\mathrm{L} 2-\mathrm{AA}$ in $\mathrm{SSrA}^{+} \mathrm{Clp}^{+}$ (SG22163) and SSrA ${ }^{+}$clpP::kan (SG22175) strains. These data were obtained by scanning of the gels shown in A and B. 


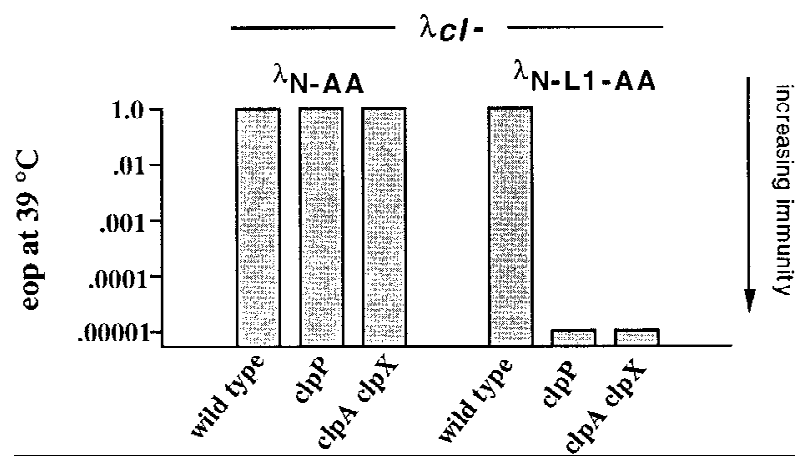

Figure 6. Phage immunity at $39^{\circ} \mathrm{C}$. Immunity to $\lambda \mathrm{Cl}^{-}$of wildtype (SG22163), clpP (SG22174), and clpA clpX (SG22178) strains expressing $\lambda \mathrm{N}-\mathrm{AA}$ and $\lambda \mathrm{N}-\mathrm{AA}$ at $39^{\circ} \mathrm{C}$. Except for temperature, the plating conditions were identical to those described in Fig. 2.

Our studies show that CIPXP and CIPAP, two related protease complexes, degrade SsrA-tagged proteins in the bacterial cytoplasm. This ATP-dependent degradation of SsrA-tagged substrates can be reproduced with purified components in vitro, and requires a substrate with a wild-type SsrA tail, the presence of both CIpP and either CIpA or CIpX. Keiler et al . (1996) showed previously that periplasmic proteins containing the SsrA-tag are degraded by Tsp (Prc), a protease that does not require or use ATP. There are, therefore, at least three proteases in E. coli, which function in the SsrA quality-control surveillance system. In addition, as discussed below, there are hints that one or more additional cytoplasmic proteases may al so degrade SsrA-tagged substrates. This indicates both that carboxy-terminal-specific substrate recognition is rel atively common among bacterial proteases and that the SsrA quality-control system has evolved to use a substantial number of different proteolytic systems.

CIpAP and CIpXP have mini-proteasome structures composed of four stacked rings (Kessel et al. 1995; Grimaud et al. 1998), in which CIpP, the proteolytic component, forms the central portion. The crystal structure of CIpP shows a double-ring assembly of heptamers in which the active sites are located in a central proteolytic chamber with small axial entrance pores (Wang et al. 1997). It has been proposed that CIpX or CIpA, which themselves form the outer rings of the proteolytic complex, act in a chaperone-like fashion to help denature substrates and feed them into the active-site chamber of ClpP (Gottesman et al. 1997; Wang et al. 1997). This model is appealing as both CIpX and CIpA, in the absence of CIpP, have been shown to have ATP-dependent chaperone activities that catalyze the disassembly of protein oligomers (Wickner et al. 1994; Levchenko et al. 1995). The ability of CIPA and CIpX to use the energy of ATP hydrolysis to aid in protein degradation may be important to allow SsrA-tagged substrates to be degraded even when these proteins are stably folded, as is the case, for example, for the SsrA-tagged amino-terminal domain variants of $\lambda$ repressor studied here.
Recognition of protein substrates by CIpXP and CIpAP is mediated by the CIpX and CIpA subunits (Gottesman et al. 1993; Wojtkowiak et al. 1993), and recent proteindissection studies have shown that CIpX contains modular domains that bind to the carboxy-terminal peptides of several target proteins, including SsrA-tagged A rc repressor (Levchenko et al. 1997). Because both CIpA and Tsp al so contain domains homologous to the substrate-binding domains of CIpX (Levchenko et al. 1997), it seems very likely that CIpXP, CIpAP, and Tsp will all recognize SsrA-tagged substrates by a common mechanism that empl oys independent peptide-binding domains to recognize the carboxy-terminal SsrA tag.

CI pAP degrades certain $\beta$-gal actosi dase fusi on proteins in vivo and degrades model peptides and casein in vitro (Gottesman et al. 1990; Tobias et al. 1991). Remarkably, the CIPAP-dependent degradation of certain of these $\beta$ galactosidase fusions is controlled by the identity of their amino-terminal amino acids (Tobias et al. 1991). Therefore, there is a somewhat startling difference between our results, which indicate carboxy-terminal recognition by the CI PAP enzyme, and those of Tobias et al. (1991), which suggest amino-terminal recognition. These apparently conflicting results might be reconciled if CIPAP contained a substrate-binding domain that recognized amino-terminal determinants in addition to the peptide-binding domain that apparently recognizes carboxy-terminal determinants.

$\mathrm{ClpX}$ recognizes the carboxy-terminal regions of several specific substrates including MuA transposase, $\mathrm{Mu}$ repressor, and certain virulent derivatives of $\mathrm{M} \mathrm{u}$ repressor (Levchenko et al. 1995; Laachouch et al. 1996; Vogel et al. 1996). Hence, the carboxy-terminal specificity shown here for CIpXP degradation of SsrA-tagged substrates is consonant with prior results. It is important to note, however, that the common specificity that we observe for CIpAP and CIpXP degradation of SsrA-tagged proteins contrasts with previous results, in which little or no cross-reactivity was observed for specific substrates (for review, see Gottesman 1996). It remains to be determined how the CIpA and CIpX enzymes recognize common SsrA-tagged substrates but have distinct specificities in other cases.

CIpXP and CIpAP must be responsible for most of the intracellular degradation of the $\lambda \mathrm{N}-\mathrm{AA}$ and $\lambda \mathrm{N}-\mathrm{L} 1-\mathrm{AA}$ proteins in $\mathrm{E}$. coli growing at $32^{\circ} \mathrm{C}$, as little residual degradation of either SsrA-tailed protein is observed in cells mutant for clpP or mutant for both clpA and clpX. Several observations, however, suggest that other cytoplasmic proteases are al so capable of degrading SsrA-tagged proteins under appropriate conditions. For example, degradation of the $\lambda \mathrm{N}-\mathrm{AA}$ protein at $39^{\circ} \mathrm{C}$ is not fully eliminated by a clpP mutation. C. Hermann and R. D'Ari (pers. comm.) have found that $\mathrm{HflB}$, a membrane-bound protease of the AAA family (T omoyasu et al. 1995), has an important role in the high-temperature degradation of $\lambda N-A A$. We note, however, that degradation of $\lambda N-L 1-$ $A A$, which has the same SsrA tag as $\lambda N-A A$, remains dependent on CIPP at both high and low temperatures. $\mathrm{HflB}$ must not, for some reason associated with the 
linker region of $\lambda \mathrm{N}-\mathrm{L}$ 1-AA, be capable of degrading this protein efficiently. These differences might arise from linker-mediated alterations in the accessibility of the SsrA tail or possibly from binding interactions of the linker regi ons that sequester the associated protein from membrane-bound HflB.

The existence of multiple proteases that can function in SsrA quality-control surveillance may be biologically important in providing increased degradation capacity, in allowing degradation of virtually any incomplete translation product that is marked for degradation by this system, in allowing degradation in different cellular compartments, or in ensuring that SsrA-tagged proteins can be degraded under a wide variety of growth conditions. Functional redundancy or overlap of this sort makes biological sense for a general quality-control system but also requires that the SsrA degradation tag be recognized as a ubiquitous signal by proteases that normally have discrete substrate preferences. How this occurs is an intriguing problem in molecular recognition.

There are also clues that SsrA RNA may influence proteolysis of tagged proteins by mechanisms in addition to directing carboxy-terminal addition of the SsrA tag to incompl ete proteins. For example, the shorter, presumably untagged species of the $\lambda \mathrm{N}$-trpAt protein is degraded faster in $\mathrm{SsrA}^{+}$strains than in SsrA ${ }^{-}$strains. M oreover, the SsrA-tagged species of $\lambda \mathrm{N}$-trpAt in which the tag is added cotranslational ly is degraded faster than $\lambda \mathrm{N}$ L2-AA, a protein with essentially the same sequence in which the SsrA tail is DN A encoded (Fig. 5). There are a number of ways to rationalize these results. SsrA recognition of stalled ribosomes might recruit proteases or degradation chaperones; the proteolytic susceptibility of proteins synthesized from mRN As without stop codons might be influenced by changes in the kinetics of translation or protein folding; and/or additional proteolytic activities may be induced in SSrA ${ }^{+}$strains.

Some of the phenotypes of ssrA mutants suggest effects beyond those caused by Clp-dependent stabilization of protein fragments. ssrA mutants grow slowly under a number of conditions (Oh and Apirion 1991; Komine et al. 1994; Trempy et al. 1994), whereas clpP mutants do not suffer the same growth problems under these conditions (S. Gottesman, unpubl.). Although this may simply indicate that degradation of some SsrAtagged proteins requi res proteases such as Tsp or $\mathrm{HflB}$, it is also possible that the relief of ribosome stalling by SsrA is as important as the subsequent degradation of proteins or that tagging of particular proteins as part of their normal synthesis is important for cell growth. It is worth pointing out that ssrA mutants have been found to have a variety of as yet unexplained phenotypes including induction of a protease activity with Lon-like specificity (Kirby et al. 1994), defects in phage P22 growth (Retallack et al. 1994), and enhancement of the activities of a variety of phage and bacterial repressors (Retallack and Friedman 1995). The extent to which these phenotypes might be independent of the degradation of SsrAtagged proteins is an important question that remains to be answered.

\section{Materials and methods}

Strains and plasmids

Hosts for experiments in vivo were a set of isogenic strains, each containing lacl $^{\mathrm{Q}}$ inserted into the malP gene. The parental strain, SG21163, is derived from SG20250 (Gottesman et al. 1985); a malP::Iacl ${ }^{\mathrm{Q}}$ derivative was constructed as described previously (Jubete et al. 1996). From SG21163, the various protease mutations were introduced by $\mathrm{PI}$ transduction and either selection for the inserted antibiotic resistance (SG21164, clpB::kan; SG21165, clpP::cat $\Delta$ Ion; SG22168, hflA::kan; SG22174, clpP::cat; SG22175, clpP::kan (polar on clpX); SG22176, clpA::kan; and SG22177, clpX::kan), linkage of Ion to proC (SG22186, $\Delta$ lon rcsA51::kan), or linkage to a marker for hflBts [SG22166, hflB1ts linked to tet, transduced from SL93, a gift from C. Herman and R. D'Ari (Herman et al. 1993)]. To construct the clpX clpA double mutant, SG22178, we first linked zba-1091::tet [from SG 1091; (M aurizi et al. 1985)] to clpX::kan and then transduced from this host to the clpA::kan strain, selecting for tetracycline resistance. Transductants were screened initially for increased immunity in the presence of the $\mathrm{p} \lambda \mathrm{N}-\mathrm{SPT}$ plasmid and subsequently checked by back transduction of the tet resistance to a kanamycin sensitive strain and demonstration of ability to transfer the linked clpX::kan marker. Strain GL005 is Ion- ${ }^{-} \mathrm{clpQ}^{-}$rcsA- $^{-}$(G. Leffers, unpubl.).

The isogenic protease-deficient strains were transformed with the plasmids shown in Figure 1 . Plasmids $p \lambda N-S P T$ and $p \lambda N$ CPT encode residues 1-93 of $\lambda$ repressor (the amino-terminal domain) with the carboxy-terminal extensions AANDENYALAA and AANDENYALDD, respectively (Keiler et al. 1996). The proteins encoded by these plasmids are referred to as $\lambda \mathrm{N}$ $A A$ and $\lambda N-D D$, respectively. Plasmids pER 130 and pER132 encode similar constructs but have a linker with an M 2 FLAG epitope and $6 \mathrm{H}$ is sequence inserted between residues 1-93 and the carboxy-terminal extensions (Fig. 1); the resulting proteins are referred to as $\lambda \mathrm{N}$-L1-AA and $\lambda \mathrm{N}$-L1-DD. To construct pER130, PCR was used to amplify the carboxy-terminal portion of a $\lambda$ repressor amino-terminal domain-M2-FLAG-6 His gene in plasmid pAD103 (A. Davidson, unpubl.) using primers ER27 (5'-TATAACGCGGCATTGCTAGCAAA-3') and ER30 (5'-CCGGATCCTTAAGCTG CTAAAGCGTAGTTTTCGTCGTTTGCTGCGTGATGGTGATGATGGTGCTTGTCA-3'). The resulting product was cut with $\mathrm{N}$ hel and $\mathrm{BamHI}$ and ligated to the pAD103 Nhel-Bglll backbone fragment. Plasmid pER132 was constructed in the same fashion using primers ER27 and ER32 (5'-CCGGATCCTTAATCGTCTAAAGCGTAGTTTTC GTCGTTTGCTGCGTGATGGTGATGATGGTGCTTGTCA-3').

The expected structure of each construct was verified by restriction mapping and DNA sequencing.

The backbones for pPW500, pER161, and pER163 (Fig. 1) are from pAD 100, a pBR322-based plasmid carrying a $P_{\text {trc }}$ promoter (trp/lacUV5 hybrid promoter), lacl $^{\mathrm{Q}}$ and an $\mathrm{F}_{1}$ phage origin (Davidson and Sauer 1994). Cloning into pAD 100 resulted in a change in the amino-terminal amino acid of $\lambda$ repressor from Ser to Gly. This sequence change, however, does not affect repressor activity (Clarke et al. 1991). Construction of pPW500, which contains a trpAt terminator following the amino-terminal domain and directs transcription of an mRNA with no in-frame stop codons, has been described previously (see footnote 14 in Keiler et al. 1996). Addition of the AANDENYALAA carboxyterminal sequence to the $\lambda \mathrm{N}$-trpAt protein is dependent on SsrA-mediated addition of the tag sequence in vivo, and therefore there can be untagged or SsrA-tagged versions of $\lambda \mathrm{N}$-trpAt. Plasmids pER161 and pER163 encode proteins ( $\lambda N$-L2-AA and $\lambda N$-L2-DD, respectively) with linkers containing the same 
amino acids as those encoded by the trpA terminator sequence. As a result, the SsrA-tagged $\lambda \mathrm{N}$-trpAt protein and the $\lambda \mathrm{N}$-L2AA protein should have the same or very similar amino-acid sequences (see Fig. 1 and legend). Plasmid pER161 was constructed by amplifying plasmid pAD 103 with primers ER60 (5' GCGCCATG GGCACAAAAAAGAAACCATTA-3') and ER61 (5'-CCGGATCCTTAAGCTGCTAA AGCGTAGTTTTCGTCGTTTGCTGCTCCGGACATTAATCGTGCGGCGTGATGGTGATGATGGTTGCTT-3'). The resulting product was cut with $\mathrm{Ncol}$ and BamHI and ligated to the pAD100 Ncol-BgllI backbone fragment. Plasmid pER 163 was constructed in the same fashion using primers pER60 and ER62 (5'-CCGGATCCTTAATCGTCTA AAGCGTAGTTTTCGTCGTTTGCTGCTCCGGACATTAATCGTGCGGCGTGATGGTGATGATGGGTGCTT-3'). The expected structure of each construct was verified by DNA sequencing.

\section{Steady-state expression and degradation in vivo}

Intracellular levels of different amino-terminal domain variants, which reflect a balance between the rates of synthesis and degradation of these proteins, were assayed by measuring immunity to superinfecting $\lambda$ phage. Cells containing high levels of the amino-terminal domain of $\lambda$ repressor are immune, whereas those containing low levels are sensitive to phage infection. Cells containing plasmids encoding $\lambda N-A A, \lambda N-D D$, $\lambda \mathrm{N}$-L1-AA, $\lambda \mathrm{N}$-L1-DD, $\lambda \mathrm{N}$-L2-AA, $\lambda \mathrm{N}$-L2-DD, and $\lambda \mathrm{N}$-trpAt were grown without IPTG induction in tryptone broth with vitamin B1, maltose, $0.01 \mathrm{M} \mathrm{M} \mathrm{gSO}_{4}$, and ampicillin $(100 \mu \mathrm{g} / \mathrm{ml})$ at $32^{\circ} \mathrm{C}$ to late log phase. Lawns of cells were plated in top agar on LB or LB-Amp plates, spotted with dilutions of $\lambda \mathrm{Cl}^{-}, \lambda \mathrm{Cl}$, $\lambda v i r$, and $\lambda i m m 21 \mathrm{cl}$ phages, and incubated at $32^{\circ} \mathrm{C}$ unless indicated. Efficiencies of plating are reported relative to growth on cells with vector alone or with no plasmid; both control strains gave identical results. The imm21cl phage plated with an efficiency of 1.0 on all hosts. $\lambda$ vir, defined as virulent by its ability to plate on normal $\lambda$ lysogens, plated on all hosts, although the eop was low or plaques were smaller in some cases. $\lambda \mathrm{Cl}^{-}$is our standard for testing immunity; it fails to plate on normal $\lambda$ lysogens. $\lambda$ c17 is a weak clear mutant and was used here because it was sensitive to intermediate levels of the amino-terminal domain of $\lambda$ repressor in initial tests. $\lambda c 17$ carries a wildtype $\mathrm{cl}$ gene but has a duplication of $9 \mathrm{bp}$ in the right operon, which creates a new, constitutively expressed rightward promoter that allows synthesis of $\lambda$ replication proteins and leads to replication and titration of repressor (Sly and Rabideau 1969; Rosenberg et al. 1978). It seems likely that $\lambda c 17$ acts as a weak clear mutation in these tests because, in the presence of even low levels of endogenous repression within the cell, the positive self-regulation of $\mathrm{P}_{\mathrm{RM}}$ will lead to synthesis of wild-type $\mathrm{Cl}$ (both stable and able to oligomerize) from the incoming c17 phage, preventing further phage growth and release of a phage burst.

Degradation of the amino-terminal domain variants in vivo was measured by Western blotting after protein synthesis was blocked by addition of spectinomycin. Cells were grown at $32^{\circ} \mathrm{C}$ in tryptone broth plus ampicillin $(100 \mu \mathrm{g} / \mathrm{ml})$ to an $A_{600}$ of 0.3 and induced for $30 \mathrm{~min}$ with IPTG (final concentration $0.02 \%$ ). A zero-time sample was removed and spectinomycin was added to a final concentration of $100 \mu \mathrm{g} / \mathrm{ml}$ to block further protein synthesis. One $\mathrm{ml}$ samples were removed periodically to tubes with $110 \mu \mathrm{l}$ of $50 \%$ TCA. After centrifugation, pellets were washed with $80 \%$ acetone, dried, and resuspended in tricine-gel loading buffer. Equal quantities of protein extracts were separated on a $15 \%$ SDS-polyacrlamide gel or $10 \%-20 \%$ tricine SDS-polyacrylamide gel (see figure legends) and transferred to nitrocellulose filters. Filters were incubated with polyclonal anti- $\lambda$-repressor antibody, a gift of Jeffrey Roberts (Cornell University, Ithaca, NY), or the M 2 monoclonal anti-FLAG antibody (Eastman Kodak). Immunobl ots were devel oped using HPR-conjugated goat anti-rabbit or anti-mouse antibody, followed by enhanced chemiluminescence (Amersham). Blots were quantified by scanning films with the Eagle Eye II gel imager (Stratagene).

\section{Purification of ${ }^{35} \mathrm{~S}$-labeled substrate proteins}

The $\lambda N$-L1-AA and $\lambda N$-LI-DD proteins were expressed in E. coli strain X90 transformed with pER130 or pER132. Cells were grown to mid-log phase at $37^{\circ} \mathrm{C}$ in $\mathrm{M} 9$ minimal medium lacking cysteine and methionine, and protein expression was induced by adding isopropyl-1-thio- $\beta$-D-gal actopyranosi de to $200 \mu \mathrm{g} / \mathrm{ml}$. Twenty minutes after induction, $\left.{ }^{35} \mathrm{~S}\right]$ methionine was added (40 $\mu \mathrm{Ci} / \mathrm{ml}$ culture). After $1 \mathrm{hr}$ at $37^{\circ} \mathrm{C}$, the cells were harvested by centrifugation and frozen at $-80^{\circ} \mathrm{C}$. Frozen cells were thawed, resuspended in Buffer $\mathrm{A}[6 \mathrm{~m}$ guanidine hydrochloride, $0.1 \mathrm{M}$ $\mathrm{NaH}_{2} \mathrm{PO}_{4}, 0.01 \mathrm{M}$ Tris ( $\mathrm{pH}$ 8.0)] containing $10 \mathrm{~mm}$ imidazole (40 $\mu \mathrm{l} / \mathrm{ml}$ culture) and al lowed to lyse while rocking at $4^{\circ} \mathrm{C}$ for $1 \mathrm{hr}$. After this time, cell debris was removed by centrifugation at 20,000g, N i-NTA agarose (Qiagen) was added (75 $\mu \mathrm{l} / \mathrm{ml}$ supernatant) and the samples were placed on a rocker for $15 \mathrm{~min}$. The resin was washed three times with buffer $A$ containing $10 \mathrm{~mm}$ imidazole, and the $\lambda N-L 1-A A$ or $\lambda N-L 1-D D$ proteins were eluted with buffer A containing $400 \mathrm{~mm}$ imidazole. Samples were dial yzed against three changes of buffer containing $50 \mathrm{~mm}$ HEPES/ NaOH (pH 7.5), and $100 \mathrm{~mm} \mathrm{NaCl}$. The purified proteins were greater than $90 \%$ pure as assayed by SDS-polyacrylamide gel electrophoresis and staining with Coomassie blue.

\section{Proteins}

Purified ClpP and 6 His-tagged ClpX were gifts from lgor Levchenko and Tania Baker (MIT, Cambridge, MA); purified CIpA was a gift from Sue Wickner (NIH, Bethesda, MD). TLCK treated $\alpha$-chymotrypsin from bovine pancreas $(60 \mathrm{U} / \mathrm{mg}$ protein) was purchased from Sigma. Unlabeled $\lambda N$-L1-AA or $\lambda N$ L1-DD were purified by denaturing nickel NTA chromatography according to the Qiagen protocol with minor modifications.

\section{Proteolytic assays in vitro}

Proteolytic digestions were performed in a total volume of $20 \mu \mathrm{l}$ in buffer containing $25 \mathrm{~mm} \mathrm{HEPES} / \mathrm{N} \mathrm{aOH}$ (pH 7.5), $25 \mathrm{~mm}$ potassium acetate, $5 \mathrm{~mm} \mathrm{M} \mathrm{gCl} 2,10 \%$ glycerol, and $0.02 \% \mathrm{NP}-40$. Complete CIpAP or CIpXP digests also contained $5 \mathrm{~mm} \mathrm{ATP,}$ $0.1 \mathrm{mg} / \mathrm{ml}$ creatine kinase, $10 \mathrm{~mm}$ creatine phosphate, 10 pmoles of ${ }^{35} \mathrm{~S}$-labeled protein substrate $(\lambda \mathrm{N}$-L1-AA or $\lambda \mathrm{N}$-L1DD), 3.5 pmoles of CIpP subunits, and 3 pmoles of CIpA or 3.5 pmoles ClpX subunits. Complete chymotrypsin digests contained 10 pmoles protein substrate and 200 ng of $\alpha$-chymotryp$\sin$. Digestion solutions were incubated at $30^{\circ} \mathrm{C}$ for different times, the reactions stopped by the addition of TCA to $10 \%$, and the tubes left on ice. After a minimum of $30 \mathrm{~min}$, the samples were centrifuged for $10 \mathrm{~min}$ at $20,000 \mathrm{~g}$ and $4^{\circ} \mathrm{C}$, and TCA soluble counts were determined by scintillation counting in Ready Safe liquid scintillation cocktail (Beckman). Control $2 \mathrm{hr}$ digests lacking either CIpX or ClpA, CIpP, or ATP showed an average of $3 \%-4 \%$ of the total ${ }^{35} \mathrm{~S}$ counts in the supernatant after TCA precipitation. These average values were subtracted as background from the complete digestion samples containing CIPAP/ATP or CIPXP/ATP. 
Circular-dichroism spectra and thermal melts

The circular-dichroism (CD) spectra of the $\lambda N$-L1-AA and $\lambda N$ L1-DD proteins were recorded at a concentration of $3.8 \mu \mathrm{M}$ in 25 $\mathrm{mm} \mathrm{HEPES} / \mathrm{N} \mathrm{aOH}$ (pH 7.5), $50 \mathrm{~mm} \mathrm{NaCl}$ at $25^{\circ} \mathrm{C}$ using an AVIV model 60DS spectropolarimeter. Thermal melts, performed using the same buffer and protein concentrations, were monitored by changes in CD ellipticity at $230 \mathrm{~nm}$. M el ts were performed in $1^{\circ} \mathrm{C}$ increments with a $42-\mathrm{sec}$ equilibration time and a $30-\mathrm{sec}$ averaging time at each temperature.

\section{Acknowledgments}

We thank Ken Keiler and Patrick Waller for strains and plasmids; Igor Levchenko, Tania Baker, and Sue Wickner for purified proteins; Jeffrey Roberts for $\lambda$ repressor antibodies; $C$. Hermann and R. D'Ari for strains and communication of unpublished results; and Tania Baker and Michael Maurizi for hel pful discussions and comments on the manuscript. This work was supported in part by the intramural program of the $\mathrm{N}$ ational Cancer Institute and by $\mathrm{N}$ ational Institutes of Heal th grant AI16897.

The publication costs of this article were defrayed in part by payment of page charges. This article must therefore be hereby marked "advertisement" in accordance with 18 USC section 1734 solely to indicate this fact.

\section{References}

Benyon, R.J. and J.S. Bond. 1989. Proteolytic enzymes: A practical approach IRL Press, Oxford, UK.

Bowie, J.U. and R.T. Sauer. 1989. Identification of C-terminal extensions that protect proteins from intracellular proteolysis. J. Biol. Chem. 264: 7596-7602.

Clarke, N.D., L.J. Beamer, H.R. Goldberg, C. Berkower, and C.O. Pabo. 1991. The DNA binding arm of $\lambda$ repressor: Critical contacts from a flexible region. Science 254: 267-270.

Davidson, A.R. and R.T. Sauer. 1994. Folded proteins occur frequently in libraries of random amino acid sequences. Proc. Natl. Acad. Sci. 91: 2146-2150.

Felden, B., H. Himeno, A. Muto, J.P. McCutcheon, J.F. Atkins, and R.F. Gesteland. 1997. Probing the structure of the Escherichia coli 10Sa RNA (tmRNA). RNA 3: 89-103.

Gottesman, S. 1996. Proteases and their targets in Escherichia coli. Annu. Rev. Genet. 30: 465-506.

Gottesman, S., P. Trisler, and A.S. Torres-Cabassa. 1985. Regulation of capsular polysaccharide synthesis in Escherichia coli K12: Characterization of three regulatory genes. J. Bacteriol. 162: 1111-1119.

Gottesman, S., W.P. Clark, and M.R. M aurizi. 1990. The ATPdependent CIp protease of Escherichia coli: Sequence of ClpA and identification of a Clp-specific substrate. J. Biol. Chem. 265: 7886-7893.

Gottesman, S., W.P. Clark, V. de Crecy-Lagard, and M.R. Maurizi. 1993. CIpX, an alternative subunit for the ATPdependent Clp protease of Escherichia coli. J. Biol. Chem. 268: 22618-22626.

Gottesman, S., S. Wickner, and M.R. Maurizi. 1997. Protein quality control: Triage by chaperones and proteases. Genes \& Dev. 11: 815-823.

Grimaud, R., M. Kessel, F. Beuron, A.C. Steven, and M.R. Maurizi. 1998. Structural and enzymatic similarities between the E. coli. ATP-dependent proteases, CIpXP and CIpAP. J. Biol. Chem. 273: (in press).

Herman, C., T. Ogura, T. Tomoyasu, S. Hiraga, Y. Akiyama, K.
Ito, R. Thomas, R. D'Ari, and P. Bouloc. 1993. Cell growth and $\lambda$ phage development controlled by the same essential Escherichia coli gene, ftsH/hflB. Proc. Natl. Acad. Sci. 90: 10861-10865.

Herman, C., D. Thévenet, R. D'Ari, and P. Bouloc. 1995. Degradation of $\sigma 32$, the heat shock regulator in Escherichia coli, is governed by HflB. Proc. Natl. Acad. Sci. 92: 3516-3520.

Jordan, S.R. and C.O. Pabo. 1988. Structure of the lambda complex at $2.5 \mathrm{~A}$ resolution: Details of the repressor-operator interactions. Science 242: 893-899.

Jubete, Y., M.R. Maurizi, and S. Gottesman. 1996. Role of the heat shock protein DnaJ in the Lon-dependent degradation of naturally unstable proteins. J. Biol. Chem. 271: 3079830803.

Kanemori, M., K. N ishihara, H. Y anagi, and T. Yura. 1997. Synergistic roles of HsIVU and other ATP-dependent proteases in controlling in vivo turnover of $\sigma 32$ and abnormal proteins in Escherichia coli. J. Bacteriol. 179: 7219-7225.

Keiler, K.C., K.R. Silver, K.M. Downard, I.A. Papayannopoulos, K. Biemann, and R.T. Sauer. 1995. C-terminal specific protein degradation: Activity and substrate specificity of the Tsp protease. Protein Sci. 4: 1507-1515.

Keiler, K.C., P.R.H. Waller, and R.T. Sauer. 1996. Role of a peptide tagging system in degradation of proteins synthesized from damaged messenger RN A. Science 271: 990-993.

Kessel, M., M.R. Maurizi, B. Kim, E. Kocsis, B.L. Trus, S.K. Singh, and A.C. Steven. 1995. Homology in structural organization between E. coli CIpAP protease and the eukaryotic 26S proteasome. J. Mol. Biol. 250: 587-594.

Khattar, M.M. 1997. Overexpression of the hsIVU operon suppresses SOS-mediated inhibition of cell division in Escherichia coli. FEBS Lett. 414: 402-404.

Kirby, J.E., J.E. Trempy, and S. Gottesman. 1994. Excision of a P4-like cryptic prophage leads to Alp protease expression in Escherichia coli. J. Bacteriol. 176: 2068-2081.

Komine, Y., M. Kitabatake, T. Y okogawa, K. Nishikawa, and H. Inokuchi. 1994. A tRNA-like structure is present in 10Sa RN A, a small stable RN A from Escherichia coli. Proc. Natl. Acad. Sci. 91: 9223-9227.

Laachouch, J.E., L. Desmet, V. Geuskens, R. Grimaud, and A. Toussaint. 1996. Bacteriophage $\mathrm{Mu}$ repressor as a target for the Escherichia coli ATP-dependent CIp protease. EMBO J. 15: 437-444.

Levchenko, I., L. Luo, and T.A. Baker. 1995. Disassembly of the $\mathrm{Mu}$ transposase tetramer by the ClpX chaperone. Genes \& Dev. 9: 2399-2408.

Levchenko, I., C.K. Smith, N.P. Walsh, R.T. Sauer, and T.A. Baker. 1997. PDZ-like domains mediate binding specificity in the $\mathrm{Clp} / \mathrm{Hsp} 100$ family of chaperones and protease regulatory subunits. Cell 91: 939-947.

Maurizi, M.R., P. Trisler, and S. Gottesman. 1985. Insertional mutagenesis of the Ion gene in Escherichia coli: Ion is dispensable. J. Bacteriol. 164: 1124-1135.

Missiakas, D., F. Schwager, J.M. Betton, C. Georgopoulos, and S. Raina. 1996. Identification and characterization of HsIV $\mathrm{HslU}$ (CIpQ ClpY) proteins involved in overall proteolysis of misfolded proteins in Escherichia coli. EMBO J. 15: 68996909.

Oh, B.-K. and D. A pirion. 1991. 10Sa RN A, a small stable RNA of Escherichia coli, is functional. Mol. Gen. Genet. 221: 5256.

Parsell, D.A., K.R. Silber, and R.T. Sauer. 1990. Carboxy-terminal determinants of intracellular protein degradation. Genes \& Dev. 4: 277-286.

Retallack, D.M. and D.I. Friedman. 1995. A role for a small stable RN A in modulating the activity of DN A-binding pro- 
teins. Cell 83: 227-235.

Retallack, D.M.L., L.L. Thompson, and D.I. Friedman. 1994. Role for 10Sa RNA in the growth of $\lambda-P 22$ hybrid phage. J. Bacteriol. 176: 2082-2089.

Rosenberg, M., D. Court, H. Shimatake, and C. Brady. 1978. The relationship between function and DNA sequence in an intercistronic regulatory region in phage $\lambda$. N ature 272: 414423.

Sauer, R.T., C.O. Pabo, B.J. Meyer, M. Ptashne, and K.C. Backman. 1979. The regulatory functions of the $\lambda$ repressor reside in the amino terminal domain. Nature 279: 396-400.

Silber, K.R. and R.T. Sauer. 1994. Deletion of the prc (tsp) gene provides evidence for additional tail-specific proteolytic activity in Escherichia coli K-12. Mol. Gen. Genet. 242: 237240.

Silber, K.R., K.C. Keiler, and R.T. Sauer. 1992. Tsp: A tail-specific protease that selectively degrades proteins with nonpoIar C-termini. Proc. Natl. Acad. Sci. 89: 295-299.

Sly, W.S. and K. Rabideau. 1969. Mechanism of $\lambda \mathrm{c} 17 \mathrm{cl}$ virulence. J. Mol. Biol. 42: 385-400.

Squires, C. and C.L. Squires, 1992. The CIp proteins: Proteolysis regulators or molecular chaperones? J. Bacteriol. 174: 10811085.

Tobias, J.W., T.E. Shrader, G. Rocap, and A. Varshavsky. 1991. The N -End rule in bacteria. Science 254: 1374-1376.

Tomoyasu, T., J. Gamer, B. Bukau, M. Kanemori, H. M ori, A.J. Rutman, A.B. Oppenheim, T. Yura, K. Yamanaka, H. Miki, S. Hiraga, and T. Ogura. 1995. Escherichia coli FtsH is a membrane-bound, ATP-dependent protease which degrades the heat-shock transcription factor $\sigma 32$. EMBO J. 14: 25512560.

Trempy, J.E., J.E. Kirby, and S. Gottesman. 1994. Alp suppression of Lon: Dependence on the slpA gene. J. Bacteriol. 176: 2061-2067.

Tu, G.-F., G.E. Reid, J.-G. Zhang, R.L. M oritz, and R.J. Simpson. 1995. C-terminal extension of truncated recombinant proteins in Escherichia coli with a 10Sa RNA decapeptide. J. Biol. Chem. 270: 9322-9326.

Vogel, J.L., V. Geuskens, L. Desmet, N .P. Higgins, and A. Toussaint. 1996. C-terminal deletions can suppress temperaturesensitive mutations and change dominance in the phage $\mathrm{Mu}$ repressor. Genetics 142: 661-672.

Wang, J., J.A. Hartling, and J.M. Flanagan. 1997. The structure of $\mathrm{ClpP}$ at $2.3 \AA$ resolution suggests a model for ATP-dependent proteolysis. Cell 91: 447-456.

Wickner, S., S. Gottesman, D. Skowyra, J. Hoskins, K. McKenney, and M.R. M aurizi, 1994. A molecular chaperone, CIpA, functions like DnaK and DnaJ. Proc. Natl. Acad. 91: 1221812222.

Williams, K.P. and D.P. Bartel. 1996. Phylogenetic analysis of tmRN A secondary structure. RNA 2: 1306-1310.

Wojtkowiak, D., C. Georgopoulos, and M. Zylicz. 1993. CIpX, a new specificity component of the ATP-dependent Escherichia coli Clp protease, is potentially involved in $\lambda$ DNA replication. J. Biol. Chem. 268: 22609-22617. 


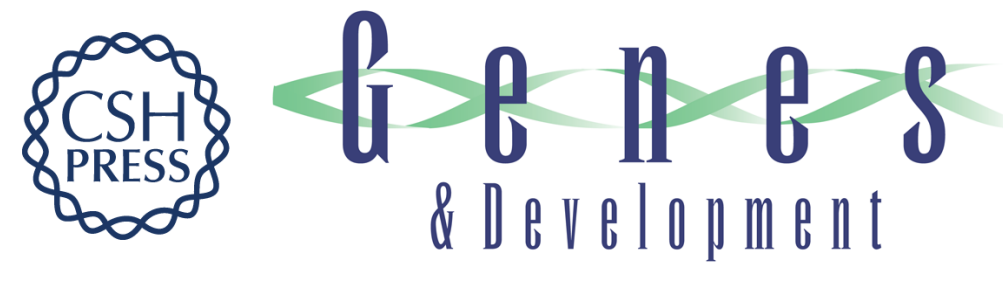

\section{The CIpXP and CIpAP proteases degrade proteins with carboxy-terminal peptide tails added by the SsrA-tagging system}

Susan Gottesman, Eric Roche, YanNing Zhou, et al.

Genes Dev. 1998, 12:

References This article cites 42 articles, 29 of which can be accessed free at:

http://genesdev.cshlp.org/content/12/9/1338.full.html\#ref-list-1

License

Email Alerting Receive free email alerts when new articles cite this article - sign up in the box at the top Service right corner of the article or click here.

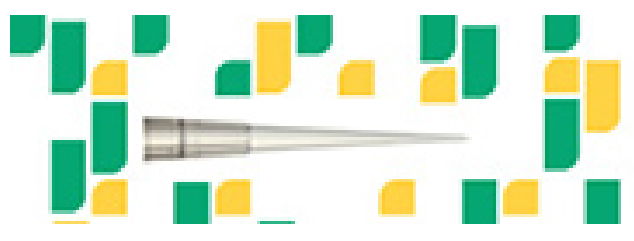

Focused on your science. 